\title{
High prevalence of Schistosoma haematobium $x$ Schistosoma bovis hybrids in schoolchildren in Cote d'Ivoire
}

Angora Etienne K. 1, 2, 3, Allienne Jean-Francois ${ }^{4}$, Rey Olivier ${ }^{4}$, Menan Herve ${ }^{3}$, Toure Andre O. 5 , Coulibaly Jean T. 1, 2, 6, 7, Raso Giovanna ${ }^{1,2}$, Yavo William ${ }^{3}$, N'Goran Eliezer K. 6, 7, Utzinger Jurg 1, ${ }^{1,}$, Balmer Oliver ${ }^{1,2}$, Boissier Jerome ${ }^{4,{ }^{*}}$

1 Swiss Trop \& Publ Hlth Inst, Basel, Switzerland.

2 Univ Basel, Basel, Switzerland.

3 Univ Felix Houphouet Boigny, Unite Format \& Rech Sci Pharmaceut \& Biol, Abidjan, Cote Ivoire.

4 Univ Perpignan Via Domitia, IFREMER, Univ Montpellier, IHPE,CNRS, Perpignan, France.

${ }^{5}$ Inst Pasteur Cote Ivoire, Abidjan, Cote Ivoire.

${ }^{6}$ Univ Felix Houphouet Boigny, Unite Format \& Rech Biosci, Abidjan, Cote Ivoire.

${ }^{7}$ Ctr Suisse Rech Sci Cote Ivoire, Abidjan, Cote Ivoire.

* Corresponding author : Jérôme Boissier, email address : boissier@univ-perp.fr

\begin{abstract}
:
Schistosomiasis is a neglected tropical disease, though it is highly prevalent in many parts of sub-Saharan Africa. While Schistosoma haematobium-bovis hybrids have been reported in West Africa, no data about Schistosoma hybrids in humans are available from Cote d'Ivoire. This study aimed to identify and quantify $\mathrm{S}$. haematobium-bovis hybrids among schoolchildren in four localities of Cote d'Ivoire. Urine samples were collected and examined by filtration to detect Schistosoma eggs. Eggs were hatched and 503 miracidia were individually collected and stored on Whatman(R) FTA cards for molecular analysis. Individual miracidia were molecularly characterized by analysis of mitochondrial cox 1 and nuclear internal transcribed spacer 2 (ITS 2) DNA regions. A mitochondrial cox1-based diagnostic polymerase chain reaction was performed on 459 miracidia, with 239 (52.1\%) exhibiting the typical band for $\mathrm{S}$. haematobium and $220(47.9 \%)$ the S. bovis band. The cox1 and ITS 2 amplicons were Sanger sequenced from 40 randomly selected miracidia to confirm species and hybrids status. Among the 33 cox1 sequences analysed, we identified $15 \mathrm{~S}$. haematobium sequences (45.5\%) belonging to seven haplotypes and $18 \mathrm{~S}$. bovis sequences (54.5\%) belonging to 12 haplotypes. Of 40 ITS 2 sequences analysed, 31 (77.5\%) were assigned to pure S. haematobium, four $(10.0 \%)$ to pure S. bovis and five (12.5\%) to S. haematobiumbovis hybrids. Our findings suggest that $\mathrm{S}$. haematobium-bovis hybrids are common in Cote d'Ivoire. Hence, intense prospection of domestic and wild animals is warranted to determine whether zoonotic transmission occurs.
\end{abstract}

Keywords : Cote d'Ivoire, hybrids, molecular analysis, Schistosoma bovis, Schistosoma haematobium 
Schistosomiasis is a parasitic disease caused by trematodes of the genus Schistosoma. It is

50 widespread in sub-Saharan Africa; yet, a key epidemiological feature of schistosomiasis is its

51 focal distribution (Colley et al., 2014; Lai et al., 2015). The disease affects more than 250

52 million people and caused an estimated 1.4 million disability-adjusted life years (DALYs) in 532017 (Hotez et al., 2014; GBD 2017 DALYs and Hale Collaborators, 2018).

In Côte d'Ivoire, urogenital and intestinal schistosomiasis, caused by Schistosoma haematobium and Schistosoma mansoni, respectively, are endemic in humans (Chammartin et al., 2014). While S. mansoni is widespread in the western part of the country (Assaré et al.,

57 2015), S. haematobium is mostly present in the central and southern parts of Côte d'Ivoire 58 (Coulibaly et al., 2013). In the northern part of Côte d'Ivoire, a recent study found a low 59 prevalence among school-aged children for both S. haematobium (1.9\%) and S. mansoni (3.5\%)

60 (M'Bra et al., 2018). Schistosoma bovis, a parasite of domestic animals, has also been reported 61 in Côte d'Ivoire, but there is a paucity of recent data. In 1997, post-mortem examinations of cattle in the savannah area of Côte d'Ivoire revealed a prevalence of 35\% (Achi et al., 2003).

Schistosoma haematobium and S. bovis are phylogenetically closely related and freshwater

64 snails of the genus Bulinus act as intermediate hosts for both species (Cook and Zumla, 2009).

The close phylogenetic association enables inter-species mating and can result in hybridization between the two species, which might influence disease transmission and alter phenotypic characteristics of parasites in both human and animal (Boissier et al., 2016; Huyse et al., 2009). 
Of particular concern, hybridization might enhance transmission and expand the distribution of schistosomiasis. For instance, laboratory hybrids exhibit particular life-history traits, including increased virulence, expansion of host spectrum, maturation and egg production (Leger and Webster, 2017). There is a need to study S. haematobium $\mathrm{x}$ S. bovis hybrids and determine how such hybrids might influence the epidemiology and control of schistosomiasis. Previous work focused on Benin, Mali, Niger and Senegal (Huyse et al., 2013; Leger and Webster, 2017). Recently, hybrids have also been involved in infections in Corsica, France (Boissier et al., 2016). While S. haematobium x S. bovis hybrids have been observed in Bulinus snails (Tian-Bi et al., 2019) in Côte d'Ivoire, no data are currently available for humans.

This study molecularly characterised schistosome miracidia collected from schoolchildren in four locations in Côte d'Ivoire to investigate the presence and extent of $S$. haematobium x $S$. bovis hybrids.

\section{Materials and methods}

\section{Ethical consideration}

Ethical clearance for this study was obtained from the Ministère de la Santé et de l'Hygiène Publique de Côte d'Ivoire (reference no. 003-18/MSHP/CNER-kp). School authorities, teachers, participating children and their parents/guardians were informed about the objectives, procedures, and potential risks and benefits of the study. Written informed consent was obtained from children's parents/guardians, while children provided oral assent.

\section{Study area}

The study was carried out in four locations of Côte d'Ivoire: (i) Agboville ( $5^{\circ} 55^{\prime} 41^{\prime \prime} \mathrm{N}$ latitude, $4^{\circ} 13^{\prime} 01^{\prime \prime} \mathrm{W}$ longitude) and (ii) Adzopé (6 $\left.6^{\circ} 06^{\prime} 25^{\prime \prime} \mathrm{N}, 3^{\circ} 51^{\prime} 36^{\prime \prime} \mathrm{W}\right)$ in the south-eastern part of the country; (iii) Sikensi $\left(5^{\circ} 40^{\prime} 34^{\prime \prime} \mathrm{N}, 4^{\circ} 34^{\prime} 33^{\prime \prime} \mathrm{W}\right)$ in the south-central part; and (iv) Duekoué $\left(6^{\circ} 44^{\prime} 00^{\prime \prime} \mathrm{N}, 7^{\circ} 21^{\prime} 00^{\prime \prime} \mathrm{W}\right)$ in the western part. The study was integrated into a cross- 
sectional survey determining the prevalence of Schistosoma infection among school-aged children (Angora et al., 2019). The four locations are well known for their high endemicity of S. haematobium (N'Guessan et al., 2007) and S. mansoni (Raso et al., 2005). Figure 1 shows the study area.

\section{Collection of miracidia}

From January to April 2018, a total of 1,187 children aged 5-14 years from the four locations (Agboville, $n=402$; Adzopé, $n=208$; Sikensi, $n=205$; and Duekoué, $n=372$ ) were invited to provide a mid-day urine sample. Urine samples were transferred to nearby health centres for parasitological examination. S. haematobium infection was identified by urine filtration (Mott et al., 1982). Ten millilitres of vigorously shaken urine was filtered through a Nytrel filter with a $40 \mu \mathrm{m}$ mesh size and examined under a microscope by experienced laboratory technicians for S. haematobium egg detection. Infection intensities were not determined.

Urine samples from 19 randomly selected infected children were chosen for further analysis in the four locations: Sikensi $(n=6)$, Agboville $(n=5)$, Duekoué $(n=5)$ and Adzopé $(n=3)$ (Table 1). Under a dissecting microscope, eggs were removed from each urine sample with an elongated Pasteur pipette and placed on a petri dish filled with fresh water to facilitate miracidial hatching. Miracidia were collected individually in $3 \mu 1$ of water using a micropipette and preserved on Whatman-FTA ${ }^{\circledR}$ cards (GE Healthcare Life Sciences; Amersham, UK), as described previously (Webster et al., 2012; Boissier et al., 2016). All samples were transferred to a laboratory in Perpignan, France pending molecular analysis.

\section{Diagnostic by polymerase chain reaction (PCR)}

Genomic DNA was extracted individually from 503 miracidia. A $2.0 \mathrm{~mm}$ disc containing the sample was removed from the FTA card with a Harris-Micro-Punch (VWR; London, UK) 
and incubated in $50 \mu \mathrm{l}$ of double distilled water for $10 \mathrm{~min}$. Water was removed and the disc incubated in $80 \mu \mathrm{l}$ of $5 \% \mathrm{Chelex}^{\circledR}$ (Bio-Rad; Hercules, USA) solution successively at $65^{\circ} \mathrm{C}$ for $30 \mathrm{~min}$ and then $99^{\circ} \mathrm{C}$ for $8 \mathrm{~min}$. Finally, $60 \mu \mathrm{l}$ of the supernatant was stored at $-20^{\circ} \mathrm{C}$ for subsequent molecular analysis.

Extracted DNAs were analysed using a rapid diagnostic mitochondrial cox1 RD-PCR in order to infer mitochondrial species designation. We employed species-specific primers to amplify a specific cox1 DNA region (differing in length) for S. bovis (260 bp), S. mansoni (215 bp) and S. haematobium (120 bp). Primers employed were a universal reverse (Shmb.R: 5'CAA GTA TCA TGA AAY ART ATR TCT AA -3') and three species-specific forward primers (Sb.F: 5'-GTT TAG GTA GTG TAG TTT GGG CTC AC -3'; Sm.F: 5'-CTT TGA TTC GTT AAC TGG AGT G -3'; and Sh.F: 5'-GGT CTC GTG TAT GAG ATC CTA TAG TTT G -3'). Each PCR was performed in a total reaction volume of $10 \mu$, comprising $2 \mu$ of DNA extract, $2 \mu$ of Green GoTaq flexi buffer 5X (Promega; Madison, USA), $0.6 \mu l$ of $25 \mathrm{mM}$ $\mathrm{MgCl}_{2}$ (Promega; Madison, USA), $0.2 \mu \mathrm{l}$ of $10 \mathrm{mM}$ dNTP mix (Promega; Madison, USA), 1 $\mu \mathrm{l}$ of 10x primer mix ( $4 \mu \mathrm{l}$ of $100 \mu \mathrm{M}$ reverse primer, $4 \mu \mathrm{l}$ of each $100 \mu \mathrm{M}$ forward primer and $84 \mu \mathrm{l}$ of doubled distilled water) and $1 \mathrm{U}$ of GoTaq Hot Start Polymerase (Promega; Madison, USA). The reaction conditions included an activation step of $95^{\circ} \mathrm{C}$ for $3 \mathrm{~min}$, followed by 45 cycles of $95^{\circ} \mathrm{C}$ for $10 \mathrm{sec}, 52^{\circ} \mathrm{C}$ for $30 \mathrm{sec}$ and $72^{\circ} \mathrm{C}$ for $10 \mathrm{sec}$, and a final extension at $72^{\circ} \mathrm{C}$ for 2 min. The cox1-PCR products were visualized on 3\% percent agarose gels stained with ethidium bromide. The prevalence of schistosomes with each mitochondrial cox1 signature was computed and stratified by study location using EpiInfo version 7 (Centers for Disease Control and Prevention; Atlanta, USA). Fisher's exact test was used and a p-value of 0.05 was considered significant. 
Cox1 and ITS2 sequencing

Based on the RD-PCR results, we randomly selected a subsample of 10 miracidia (five S. haematobium cox 1 and five $S$. bovis cox 1) per study location. Overall, 40 miracidia were sequenced on both mitochondrial $\operatorname{cox} 1$ and nuclear ITS2 gene using the following primers: Cox1.R: 5'-TAA TGC ATM GGA AAA AAA CA -3' and Cox1.F: 5'-TCT TTR GAT CAT AAG CG -3' for cox1 (Lockyer et al., 2003) and ITS 5.R: 5'-GGA AGT AAA AGT CGT AAC AAG G -3' and ITS4.F: 5'-TCC TCC GCT TAT TGA TAT GC -3' for ITS2 (Barber et al., 2000). The PCRs were performed in a final reaction volume of $25 \mu \mathrm{l}$, comprising $4 \mu \mathrm{l}$ of DNA template, $5 \mu$ l of $5 X$ Colorless GoTaq ${ }^{\circledR}$ Flexi Buffer (Promega; Madison, USA), $1.5 \mu$ of $\mathrm{MgCl}_{2}(25 \mathrm{mM}), 0.5 \mu \mathrm{l}$ of dNTP $(10 \mathrm{mM}), 0.8 \mu$ l of each $10 \mu \mathrm{M}$ primer and $0.2 \mu$ l of Go taq ${ }^{\circledR} \mathrm{G} 2$ Hot Start Polymerase (Promega; Madison, USA). The PCR conditions were the same for both markers: 3 min at $95^{\circ} \mathrm{C}$, followed by 45 cycles at $95^{\circ} \mathrm{C}$ for $40 \mathrm{sec}, 48^{\circ} \mathrm{C}$ for $40 \mathrm{sec}$ and $72^{\circ} \mathrm{C}$ for $70 \mathrm{sec}$, followed by a final extension of $2 \mathrm{~min}$ at $72^{\circ} \mathrm{C}$. The mitochondrial cox 1 and nuclear ITS2 PCR products $(4 \mu \mathrm{l})$ were visualized on $1.5 \%$ percent agarose gels stained with ethidium bromide to verify band size (expected size 1,200 bp) and quality of the amplicons. All successfully amplified PCR products were purified and sequenced with the Cox1.R: 5'-TAA TGC ATM GGA AAA AAA CA -3' or the ITS4.F: 5'-TCC TCC GCT TAT TGA TAT GC 3' primers, respectively, on an Applied Biosystems Genetic Analyser at Genoscreen (Lille, France).

\section{Sequences analysis}

The partial cox 1 and ITS2 sequences were assembled separately and edited using Sequencher version 4.5 (Gene Codes Corporation; Ann Arbor, USA). All sequences were aligned using BioEdit version 7.0.9 (Ibis Therapeutic; Carlsbad, USA) and compared to sequences deposited in the GenBank Nucleotide Database. The nuclear-ITS2 region differs at five polymorphic sites between S. haematobium and S. bovis, and hence, the sequence 
chromatograms were checked at these mutation points to identify possible heterogeneity, as previously described (Webster et al., 2013). The mitochondrial-cox1 haplotype and nucleotide diversities ( \pm standard deviation $[\mathrm{SD}]$ ) were calculated using DnaSP version 6.0 (Rozas et al., 2017). Phylogenetic trees were constructed separately for S. haematobium and S. bovis cox 1 haplotypes using MEGA version 6.0.6 (Penn State University; Pennsylvania, USA) and employing a maximum likelihood and the HKY + G nucleotide substitution model, which was determined by MEGA version 6.0.6 (Penn State University; Pennsylvania, USA) as the model best describing the data. The support for tree nodes was calculated with 1,000 bootstrap iterations. The phylogenies include all the haplotypes identified in this study plus some reference haplotypes obtained from GenBank Nucleotide Database. The phylogeny of the S. bovis cox1 data was rooted with a S. haematobium haplotype (JQ397330.1) and the S. haematobium cox1 data with a S. bovis haplotype (AJ519521.1). All cox1 sequences were uploaded onto the GenBank Nucleotide Database (GenBank accession nos. MK757162MK757168 for S. haematobium and MK757170- MK757181 for S. bovis).

\section{Results}

\section{Cox1 rapid diagnostic $P C R$}

Of the 1,187 urine samples examined, $166(14.0 \%)$ were found positive for Schistosoma eggs, as described elsewhere (Angora et al., 2019). Overall, 503 miracidia were collected from hatched eggs of 19 Schistosoma-infected children on Whatman ${ }^{\circledR}$ FTA cards and the cox1 RDPCR was successful for 459 miracidia. Of these, 239 miracidia (52.1\%) gave a S. haematobium cox1 profile and $220(47.9 \%)$ gave a $S$. bovis cox 1 profile, with no statistically significant difference between the two proportions $(\mathrm{p}=0.081$ ). No miracidia gave a $S$. mansoni cox 1 profile. The $S$. haematobium cox 1:S. bovis cox 1 ratio varied according to study area (76:63 for Sikensi; 73:38 for Agboville; 63:65 for Duekoué; and 27:54 for Adzopé). The proportion of S. haematobium cox 1 was higher than that of $S$. bovis $\operatorname{cox} 1$ in Sikensi $(\mathrm{p}=0.026)$ and Agboville 
$(\mathrm{p}<0.001)$, whereas $S$. bovis cox1 was the predominant species found in Adzopé $(\mathrm{p}<0.001)$. In Duekoué, S. haematobium cox1 and S. bovis cox1 were equally distributed (Figure 1). Table 1 shows the proportion of $S$. haematobium cox 1 and $S$. bovis cox 1 from the 459 cox1 RD-PCR miracidia, stratified by study location at the unit of the child.

\section{Coxl and ITS2 sequence analysis}

A total of 40 miracidia were sequenced. Good quality ITS2 sequences were obtained for all samples while, for cox1, only 33 samples yielded good sequences. Among the 33 cox1 sequences, we identified 15 S. haematobium sequences (Table 1) belonging to seven distinct haplotypes with a very low diversity showing few single nucleotide polymorphisms (SNPs) (Supplementary file 1: Table S1) and 18 S. bovis sequences belonging to 12 distinct haplotypes (Supplementary file 2: Table S2). Haplotype diversity $( \pm \mathrm{SD})$ was $0.922 \pm 0.051$ and $0.724 \pm 0.121$ for $S$. bovis and $S$. haematobium, respectively. Nucleotide diversity $( \pm \mathrm{SD})$ was $0.0094 \pm 0.0013$ and $0.0011 \pm 0.0003$ for S. bovis and S. haematobium, respectively. Among the ITS2 sequences, 31 gave a S. haematobium profile and four a S. bovis profile. Five miracidia gave double chromatogram peaks at the polymorphic positions between S. haematobium and S. bovis, suggesting heterozygosity (Huyse et al., 2009; Webster et al., 2013).

\section{Miracidia identified as hybrids and non-hybrids}

Analysis of both cox1 and ITS2 sequences together showed that $S$. haematobium $\mathrm{x}$ S. bovis miracidia were present in all the four study locations and were excreted by 12 children. Among these 12 children, nine excreted S. haematobium $\mathrm{x}$ S. bovis hybrids and seven excreted pure S. haematobium. Two children excreted both S. haematobium x S. bovis hybrids and pure S. haematobium miracidia, while one child excreted both $S$. haematobium x S. bovis hybrids and pure $S$. bovis miracidium (Table 1). Seventeen parasites were pure (16 S. haematobium and one S. bovis) and 23 were hybrids (57.5\%). Among the six S. haematobium x S. bovis 
combinations possible with our two markers (two possibilities in the haploid cox1 [S. haematobium cox1; S. bovis cox1] and three possibilities in the diploid ITS2 [S. haematobium ITS2; mixed S. haematobium ITS2-S. bovis ITS2; S. bovis ITS2]), four combinations indicate hybrids [S. bovis cox1 x S. haematobium ITS2; S. bovis cox1 x S. haematobium ITS2-S. bovis ITS2; S. haematobium cox1 x S. haematobium ITS2-S. bovis ITS2; S. haematobium cox1 x S. bovis ITS2]. Most of the hybrid profiles found were $S$. bovis cox1 x S. haematobium ITS2. Hybrids occurred at similar frequencies in all age classes.

Coxl phylogenies

Figures 2 and 3 show phylogenies of all S. haematobium and S. bovis cox 1 haplotypes, respectively. Note that S. bovis reference sequences used were obtained from animals. All S. haematobium cox1 haplotypes from Côte d'Ivoire cluster with group 1, as defined by Webster et al. (2012). Cox1 S. bovis haplotypes were split into two clusters separating those from Duekoué (Sb5-8) from those from the three remaining locations (Sb1-4 and Sb9-12).

\section{Discussion}

Hybridization of certain parasites is an emerging public health concern at the interface of infectious disease biology and evolution (King et al., 2015). We have identified $47.9 \%$ of S. bovis cox 1 profile by RD-PCR and $57.5 \%$ of $S$. haematobium $\times$ S. bovis hybrids using both $\operatorname{cox} 1$ and ITS2 sequences of miracidia collected in urine samples obtained from schoolchildren in Côte d'Ivoire. The analysis of mitochondrial cox1 gene sequences showed seven haplotypes for S. haematobium and 12 for S. bovis, which demonstrates the existence of a mitochondrial introgressive hybridization of S. haematobium cox1 by S. bovis. Similar results have been reported in Corsica (Moné et al., 2015; Boissier et al., 2016). Our findings were corroborated by analysis of the nuclear ITS2 region. 
The polymorphism analysis of the cox 1 gene shows that $S$. bovis is more polymorphic than

S. haematobium. This result is consistent with a recent microsatellite-based population genetic study in Cameroon, which reported higher gene diversity and higher allelic diversity for $S$. bovis compared to S. haematobium (Djuikwo-Teukeng et al., 2019). Of note, the cited Cameroon study compared the diversity of $S$. bovis to previous data for $S$. haematobium obtained from Niger and Zanzibar (Webster et al., 2015). The low polymorphism of S. haematobium cox1 is in line with previous studies (Webster et al., 2012; Webster et al., 2013; Gower et al., 2013). Results obtained at a regional scale (i.e. in different countries) corroborate our results from a finer spatial scale (i.e. four sites within a single country).

S. haematobium is known to be weakly structured (Webster et al., 2012). It has been shown that two groups can be identified across the parasite's range in sub-Saharan Africa: "group 1" clusters parasites from mainland Africa, while "group 2" clusters parasites exclusively from the Indian Ocean islands and the neighbouring African coastal regions (Webster et al., 2012). As expected, S. haematobium haplotypes from our study cluster with "group 1". Our study also shows that for S. bovis, there is heterogeneity in the distribution of haplotypes across the country with the haplotypes from Duekoue in the western part differentiated from those from the southern part of Côte d'Ivoire. Furthermore, the current study shows that S. haematobium x S. bovis hybrids occurred in schoolchildren from each of the four study locations.

No $S$. haematobium x S. mansoni hybrids were identified, even though such hybrids have been shown in a migrant boy from Côte d'Ivoire upon examination in France (Le Govic et al., 2019). Recently, it has been shown that Bulinus snails from the northern and central parts of Côte d'Ivoire were infected with S. bovis, S. haematobium and/or S. bovis x S. haematobium hybrids (Tian-Bi et al., 2019). The authors showed that $S$. bovis was particularly prevalent in Bulinus truncatus, S. haematobium was most prevalent in B. globosus and S. haematobium $\mathrm{x}$ S. bovis hybrids infected the two Bulinus species similarly. S. bovis-infected Bulinus were predominantly found in the northern part, while S. haematobium and hybrid-infected snails 
were mainly found in the central part of Côte d'Ivoire. These results show the importance of snail's involvement in the transmission of S. haematobium $\mathrm{x}$ S. bovis hybrids.

Most of the hybrids in our study showed cox1 sequences from $S$. bovis and nuclear ITS2 sequences from S. haematobium. This type of hybrid is the most common hybrid reported in, including cercariae collected from infected snails in Côte d'Ivoire (Tian-Bi et al., 2019), miracidia collected from infected patients in Senegal (Huyse et al., 2009) and miracidia collected during a recent schistosomiasis outbreak on Corsica (Boissier et al., 2016).

The current study found that some children excreted both pure S. haematobium and S. haematobium $\mathrm{x}$ S. bovis hybrids, which is in line with observations in Senegal (Huyse et al., 2009; Webster et al., 2013). Interestingly, we have also observed a single miracidium with a "pure" S. bovis signature (S. bovis ITS2 and S. bovis cox1) in one child, suggesting that this patient may be infected with $S$. bovis, which is traditionally considered a parasite of bovines. Such a "pure" S. bovis has been reported in eggs recovered from humans in Corsica (Boissier et al., 2016). These accounts suggest that zoonotic transmission might occur. However, additional research is needed to confirm this speculation. We assume that the genome of S. bovis is strongly introgressed, and hence, it is plausible that we may have missed signatures of $S$. haematobium ancestry due to the standard analyses performed (Webster et al., 2013). A broader coverage of the genome would no doubt identify even more hybrids and would allow a clearer distinction between "pure" parasites of each species and different levels of introgression.

\section{Conclusion}

Our study has shown that S. haematobium $\mathrm{x}$ S. bovis hybrids are common in Schistosoma egg-positive children in Côte d'Ivoire. Our observations are relevant because hybrid parasites could affect transmission dynamics, treatment efficacy and morbidity, which might jeopardize control of, and progress towards, elimination of schistosomiasis. Our findings are relevant as 
the presence of hybrids calls into question our present understanding of parasite transmission and host ranges, which in turn may affect the effectiveness of current control strategies. Intensive prospection of domestic and wild animals is warranted to determine whether real zoonotic transmission occurs.

\section{Supplementary files}

Supplementary file 1: Table S1: Nucleotide sequence at the five polymorphic sites within the 1 $200 \mathrm{bp}$ cox 1 sequences of the different S. haematobium haplotypes found using haplotype Sh1 as reference. $\mathrm{n}$, number of miracidia with the respective haplotypes. (DOCX $15 \mathrm{~KB}$ )

Supplementary file 2: Table S2: Nucleotide sequence at the 27 polymorphic sites within the 1 $200 \mathrm{bp}$ cox1 sequences of the different S. bovis haplotypes found, using haplotype Sb1 as reference. n, number of miracidia with the respective haplotype. (DOCX $21 \mathrm{~KB}$ )

Contributions of authors. EKA, HM, OB and JB conceived and designated the study. EKA, OR and JB wrote the first draft of the manuscript. EKA and JFA performed the molecular analyses. EKA and JB performed statistical analysis. OB, AOT, JTC, WY, JU and OB revised the manuscript. All authors read and approved the final manuscript prior to submission.

\section{Conflict of interest. None.}

Acknowledgements. We are grateful to the district health and village authorities of the four study areas for their support and facilitating the implementation of this study. We also thank the participants (children and their parents/guardians) for their commitment throughout the study. This work was financially supported by the Agence National de Recherche (ANR), which supports the Hybrid Swarm project (HySWARM) in Perpignan that is set within the 
frame of the "Laboratoire d'Excellence (LABEX)" TULIP (ANR-10-LABX-41). Additional financial support was obtained by the Swiss National Science Foundation for EKA, JU and OB (grant no. 31003A_170113). EKA is a recipient of a Swiss Government Excellence Scholarship (ESKAS, grant no. 2017-0746) for which he is deeply grateful.

Ethical standards. Ethical clearance for this study was obtained from the Ministère de la Santé et de l'Hygiène Publique de Côte d'Ivoire (reference no. 003-18/MSHP/CNER-kp). Written informed consent was obtained from children's parents or legal guardians. Oral assent was obtained from children. 


\section{References}

Achi, Y. L., Zinsstag, J., Yéo, N., Dea, V. and Dorchies, P. H. (2003). Les nématodes gastrointestinaux des bovins de la région des savanes de la Côte-d'Ivoire: enquête d'abattoir. Revue de Médecine Vétérinaire 154, 105-112.

Angora, E. K., Boissier, J., Menan, H., Rey, O., Tuo, K., Touré, A. O., Coulibaly, J. T., Méité, A., Raso, G., N'Goran, E. K., Utzinger, J. and Balmer, O. (2019). Prevalence and risk factors for schistosomiasis among schoolchildren in two settings of Côte d'Ivoire. Tropical Medicine and Infectious Disease 4, 110. doi: 10.3390/tropicalmed4030110.

Assaré, R. K., Lai, Y.-S., Yapi, A., Tian-Bi, Y.-N. T., Ouattara, M., Yao, P. K., Knopp, S., Vounatsou, P., Utzinger, J. and N'Goran, E. K. (2015). The spatial distribution of Schistosoma mansoni infection in four regions of western Côte d'Ivoire. Geospatial Health 10, 345. doi: 10.4081/gh.2015.345.

Barber, K. E., Mkoji, G. M. and Loker, E. S. (2000). PCR-RFLP analysis of the ITS2 region to identify Schistosoma haematobium and S. bovis from Kenya. American Journal of Tropical Medicine and Hygiene 62, 434-440.

Boissier, J., Grech-Angelini, S., Webster, B. L., Allienne, J.-F., Huyse, T., Mas-Coma, S., Toulza, E., Barré-Cardi, H., Rollinson, D., Kincaid-Smith, J., Oleaga, A., Galinier, R., Foata, J., Rognon, A., Berry, A., Mouahid, G., Henneron, R., Moné, H., Noel, H. and Mitta, G. (2016). Outbreak of urogenital schistosomiasis in Corsica (France): an epidemiological case study. Lancet Infectious Diseases 16, 971-979. doi: 10.1016/S1473-3099(16)00175-4.

Chammartin, F., Houngbedji, C. A., Hürlimann, E., Yapi, R. B., Silué, K. D., Soro, G., Kouamé, F. N., N'Goran, E. K., Utzinger, J., Raso, G. and Vounatsou, P. (2014). Bayesian risk mapping and model-based estimation of Schistosoma haematobium-Schistosoma mansoni codistribution in Côte d'Ivoire. PLoS Neglected Tropical Diseases 8, e3407. doi: 10.1371/journal.pntd.0003407.

Colley, D. G., Bustinduy, A. L., Secor, W. E. and King, C. H. (2014). Human schistosomiasis. Lancet 383, 2253-2264. doi: 10.1016/S0140-6736(13)61949-2.

Cook, G. C. and Zumla, A. (2009). Manson's Tropical Diseases. Elsevier Health Sciences.

Coulibaly, J. T., N'Gbesso, Y. K., N'Guessan, N. A., Winkler, M. S., Utzinger, J. and N'Goran, E. K. (2013). Epidemiology of schistosomiasis in two high-risk communities of south Côte d'Ivoire with particular emphasis on pre-school-aged children. American Journal of Tropical Medicine and Hygiene 89, 32-41. doi: 10.4269/ajtmh.12-0346.

Djuikwo-Teukeng, F. F., Kouam Simo, A., Allienne, J.-F., Rey, O., Njayou Ngapagna, A., Tchuem-

Tchuenté, L. A. and Boissier, J. (2019). Population genetic structure of Schistosoma bovis in Cameroon. Parasites \& Vectors 12, 56. doi: 10.1186/s13071-019-3307-0.

GBD 2017 DALYs and Hale Collaborators (2018). Global, regional, and national disability-adjusted life-years (DALYs) for 359 diseases and injuries and healthy life expectancy (HALE) for 195 
countries and territories, 1990-2017: a systematic analysis for the Global Burden of Disease study 2017. Lancet 392, 1736-1788. doi: 10.1016/S0140-6736(18)32203-7.

Gower, C. M., Gouvras, A. N., Lamberton, P. H. L., Deol, A., Shrivastava, J., Mutombo, P. N., Mbuh, J. V., Norton, A. J., Webster, B. L., Stothard, J. R., Garba, A., Lamine, M. S., Kariuki, C., Lange, C. N., Mkoji, G. M., Kabatereine, N. B., Gabrielli, A. F., Rudge, J. W., Fenwick, A., Sacko, M., Dembelé, R., Lwambo, N. J. S., Tchuem Tchuenté, L.-A., Rollinson, D. and Webster, J. P. (2013). Population genetic structure of Schistosoma mansoni and Schistosoma haematobium from across six sub-Saharan African countries: implications for epidemiology, evolution and control. Acta Tropica 128, 261-274. doi: 10.1016/j.actatropica.2012.09.014

Hotez, P. J., Alvarado, M., Basáñez, M.-G., Bolliger, I., Bourne, R., Boussinesq, M., Brooker, S. J., Brown, A. S., Buckle, G., Budke, C. M., Carabin, H., Coffeng, L. E., Fèvre, E. M., Fürst, T., Halasa, Y. A., Jasrasaria, R., Johns, N. E., Keiser, J., King, C. H., Lozano, R., Murdoch, M. E., O'Hanlon, S., Pion, S. D. S., Pullan, R. L., Ramaiah, K. D., Roberts, T., Shepard, D. S., Smith, J. L., Stolk, W. A., Undurraga, E. A., Utzinger, J., Wang, M., Murray, C. J. L. and Naghavi, M. (2014). The Global Burden of Disease study 2010: interpretation and implications for the neglected tropical diseases. PLoS Neglected Tropical Diseases 8, e2865. doi: 10.1371/journal.pntd.0002865.

Huyse, T., Webster, B. L., Geldof, S., Stothard, J. R., Diaw, O. T., Polman, K. and Rollinson, D. (2009). Bidirectional introgressive hybridization between a cattle and human schistosome species. PLoS Pathogens 5, e1000571. doi: 10.1371/journal.ppat.1000571.

Huyse, T., Broeck, F. V. den, Hellemans, B., Volckaert, F. a. M. and Polman, K. (2013). Hybridisation between the two major African schistosome species of humans. International Journal for Parasitology 2013, 687-689. doi: 10.1016/j.jpara.2013.04.001.

King, K. C., Stelkens, R. B., Webster, J. P., Smith, D. F. and Brockhurst, M. A. (2015) Hybridization in parasites: consequences for adaptive evolution, pathogenesis, and public health in a changing world. PLoS Pathogens 11, e1005098. doi: 10.1371/journal.ppat.1005098.

Lai, Y.-S., Biedermann, P., Ekpo, U. F., Garba, A., Mathieu, E., Midzi, N., Mwinzi, P., N'Goran, E. K., Raso, G., Assaré, R. K., Sacko, M., Schur, N., Talla, I., Tchuenté, L.-A. T., Touré, S., Winkler, M. S., Utzinger, J. and Vounatsou, P. (2015). Spatial distribution of schistosomiasis and treatment needs in sub-Saharan Africa: a systematic review and geostatistical analysis. Lancet Infectious Diseases 15, 927-940. doi: 10.1016/S14733099(15)00066-3

Le Govic, Y., Kincaid-Smith, J., Allienne, J.-F., Rey, O., de Gentile, L. and Boissier, J. (2019). Schistosoma haematobium-Schistosoma mansoni hybrid parasite in migrant boy, France, 2017. Emerging Infectious Diseases 25, 365-367. doi: 10.3201/eid2502.172028. 
Leger, E. and Webster, J. P. (2017). Hybridizations within the genus Schistosoma: implications for evolution, epidemiology and control. Parasitology 144, 65-80. doi: 10.1017/S0031182016001190.

Lockyer, A. E., Olson, P. D., Ostergaard, P., Rollinson, D., Johnston, D. A., Attwood, S. W., Southgate, V. R., Horak, P., Snyder, S. D., Le, T. H., Agatsuma, T., McManus, D. P., Carmichael, A. C., Naem, S. and Littlewood, D. T. J. (2003). The phylogeny of the Schistosomatidae based on three genes with emphasis on the interrelationships of Schistosoma Weinland, 1858. Parasitology 126, 203-224.

M'Bra, R. K., Kone, B., Yapi, Y. G., Silué, K. D., Sy, I., Vienneau, D., Soro, N., Cissé, G. and Utzinger, J. (2018). Risk factors for schistosomiasis in an urban area in northern Côte d'Ivoire. Infectious Diseases of Poverty 7, 47. doi: 10.1186/s40249-018-0431-6.

Moné, H., Holtfreter, M. C., Allienne, J.-F., Mintsa-Nguéma, R., Ibikounlé, M., Boissier, J., Berry, A., Mitta, G., Richter, J. and Mouahid, G. (2015). Introgressive hybridizations of Schistosoma haematobium by Schistosoma bovis at the origin of the first case report of schistosomiasis in Corsica (France, Europe). Parasitology Research 114, 4127-4133. doi: 10.1007/s00436-015-4643-4.

Mott, K. E., Baltes, R., Bambagha, J. and Baldassini, B. (1982). Field studies of a reusable polyamide filter for detection of Schistosoma haematobium eggs by urine filtration. Tropenmedizin und Parasitologie 33, 227-228.

N'Guessan, N., Acka, C. A., Utzinger, J. and N'Goran, E. K. (2007). Identification of high risks regions of schistosomiasis in Côte d'lvoire. Bulletin de la Société de Pathologie Exotique 100, $119-123$.

Raso, G., Matthys, B., N'Goran, E. K., Tanner, M., Vounatsou, P. and Utzinger, J. (2005). Spatial risk prediction and mapping of Schistosoma mansoni infections among schoolchildren living in western Côte d'Ivoire. Parasitology 131, 97-108.

Rozas, J., Ferrer-Mata, A., Sánchez-DelBarrio, J. C., Guirao-Rico, S., Librado, P., Ramos-Onsins, S. E. and Sánchez-Gracia, A. (2017). DnaSP 6: DNA sequence polymorphism analysis of large data sets. Molecular Biology and Evolution 34, 3299-3302. doi: 10.1093/molbev/msx248.

Soumahoro, M., Bosson-Vanga, A., Coulibaly, K., Abbes, S., Angora, E., Kouadio, K., N'Douba, A. K., Sissoko, D. and Dosso, M. (2014). Investigation d'un foyer épidémique de bilharziose urinaire dans l'école primaire du village de Guébo 2, Abidjan, Côte d'Ivoire. Bulletin de la Société de Pathologie Exotique 107, 185-187. doi: 10.1007/s13149-014-0362-9.

Tian-Bi, Y.-N. T., Webster, B., Konan, C. K., Allan, F., Diakité, N. R., Ouattara, M., Salia, D., Koné, A., Kakou, A. K., Rabone, M., Coulibaly, J. T., Knopp, S., Meïté, A., Utzinger, J., N'Goran, E. K. and Rollinson, D. (2019). Molecular characterization and distribution of Schistosoma cercariae collected from naturally infected bulinid snails in northern and central Côte d'Ivoire. Parasites \& Vectors 12, 117. doi: 10.1186/s13071-019-3381-3. 
Webster, B. L., Emery, A. M., Webster, J. P., Gouvras, A., Garba, A., Diaw, O., Seye, M. M., Tchuente, L. A. T., Simoonga, C., Mwanga, J., Lange, C., Kariuki, C., Mohammed, K. A., Stothard, J. R. and Rollinson, D. (2012). Genetic diversity within Schistosoma haematobium: DNA barcoding reveals two distinct groups. PLoS Neglected Tropical Diseases 6, e1882. doi: 10.1371/journal.pntd.0001882.

Webster, B. L., Diaw, O. T., Seye, M. M., Webster, J. P. and Rollinson, D. (2013). Introgressive hybridization of Schistosoma haematobium group species in Senegal: species barrier break down between ruminant and human schistosomes. PLoS Neglected Tropical Diseases 7, e2110. doi: 10.1371/journal.pntd.0002110.

Webster, B. L., Rabone, M., Pennance, T., Emery, A. M., Allan, F., Gouvras, A., Knopp, S., Garba, A., Hamidou, A. A., Mohammed, K. A., Ame, S. M., Rollinson, D. and Webster, J. P. (2015). Development of novel multiplex microsatellite polymerase chain reactions to enable high-throughput population genetic studies of Schistosoma haematobium. Parasites \& Vectors 8, 432. doi: 10.1186/s13071-015-1044-6. 


\section{Figure legends}

460

Fig. 1. Map of the four study locations in Côte d'Ivoire showing the distribution and the proportion of Schistosoma haematobium cox1 and Schistosoma bovis cox1 identified by RDPCR.

464

Fig. 2. Majority rule consensus tree from maximum likelihood analysis of 1,200 bp 466 mitochondrial cox1 sequences for Schistosoma haematobium haplotypes Sh1-Sh7 and sequences downloaded from GenBank. Groups 1 and 2 indicate the major clades defined by Webster et al. (2012). Clade support values for each node are maximum parsimony bootstrap percentages.

Fig. 3. Majority rule consensus tree from maximum likelihood analysis of $1,200 \mathrm{bp}$ mitochondrial DNA cox1 sequences for Schistosoma bovis haplotypes Sb1-Sb12 and sequences downloaded from GenBank. There are two clusters of S. bovis haplotypes: Duekoué (Sb5-Sb8) and the three other study sites (Sb1-Sb4 and Sb9-Sb12). Clade support values for each node are maximum parsimony bootstrap percentages. S. bovis reference sequences come from parasites collected from animals. 


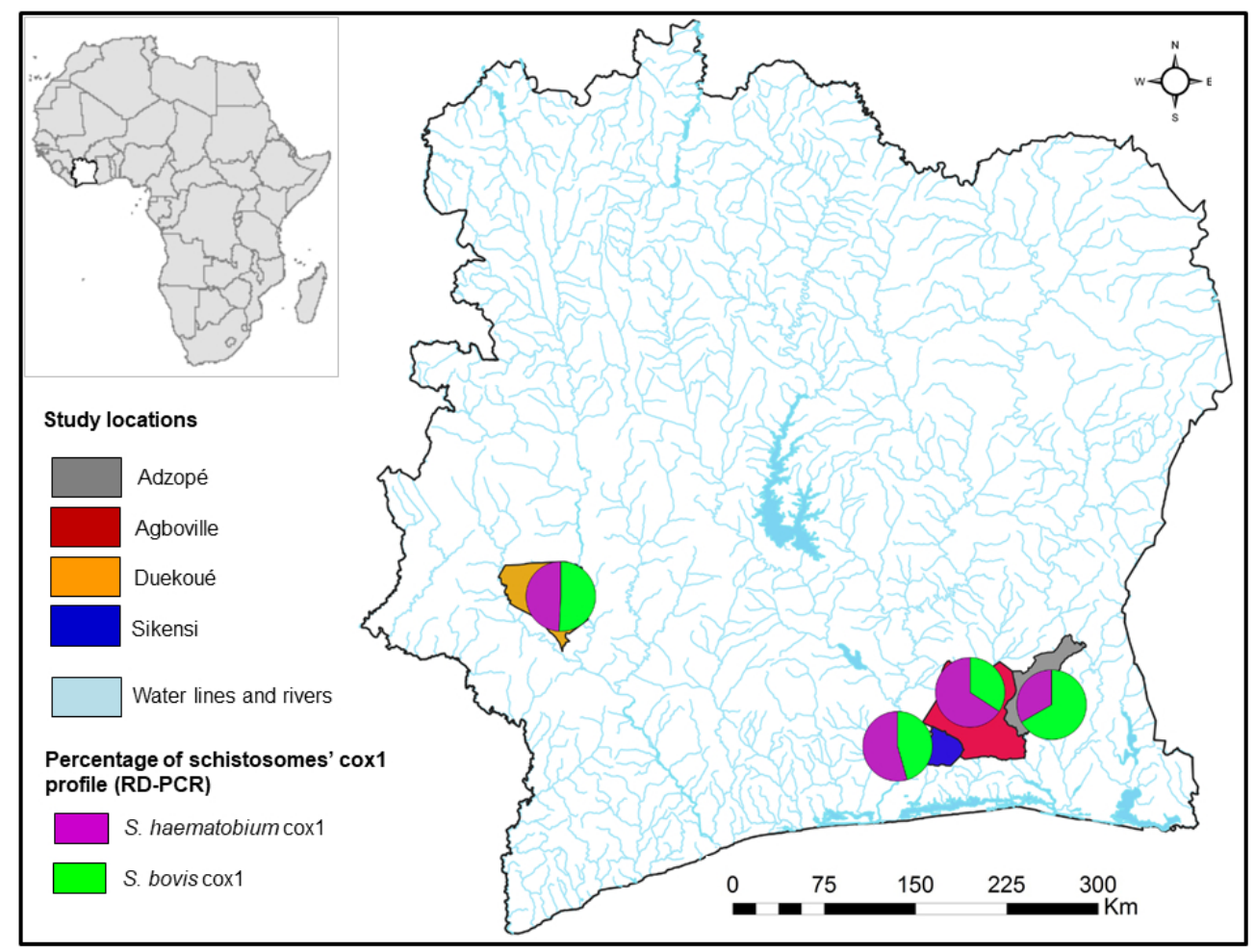

$254 \times 190 \mathrm{~mm}(96 \times 96 \mathrm{DPI})$ 


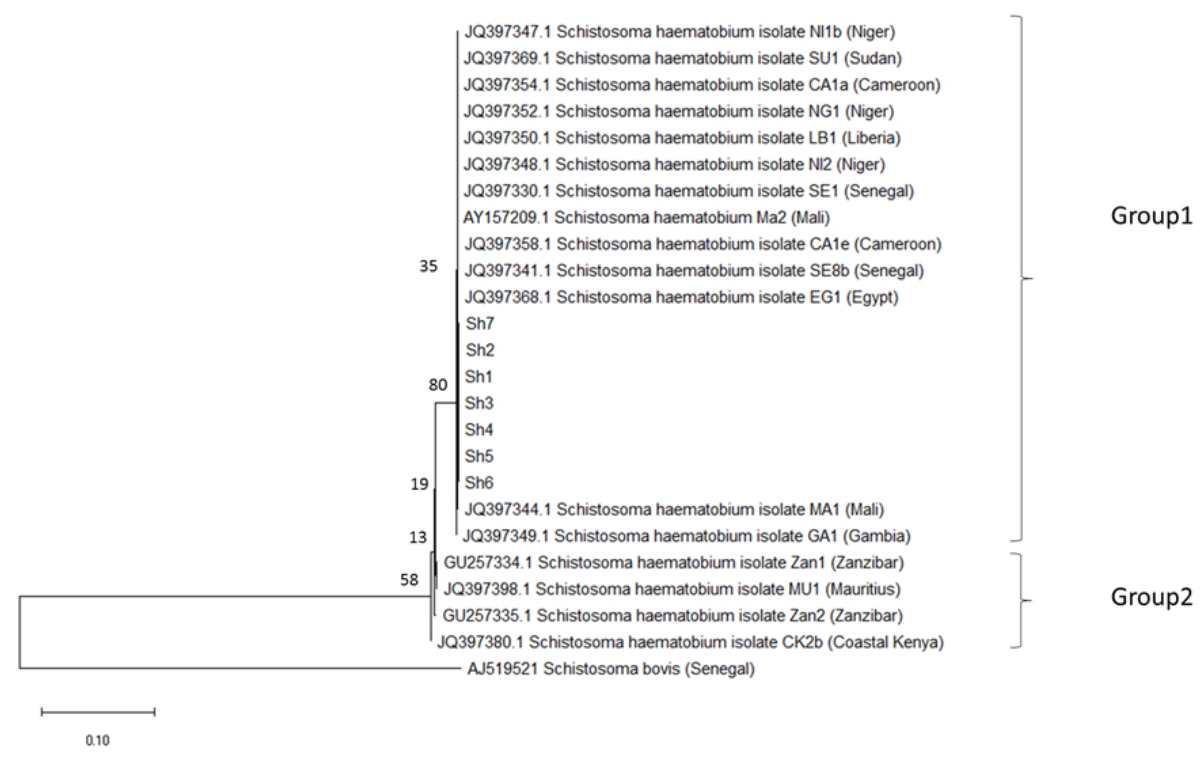

$254 \times 190 \mathrm{~mm}(96 \times 96$ DPI) 


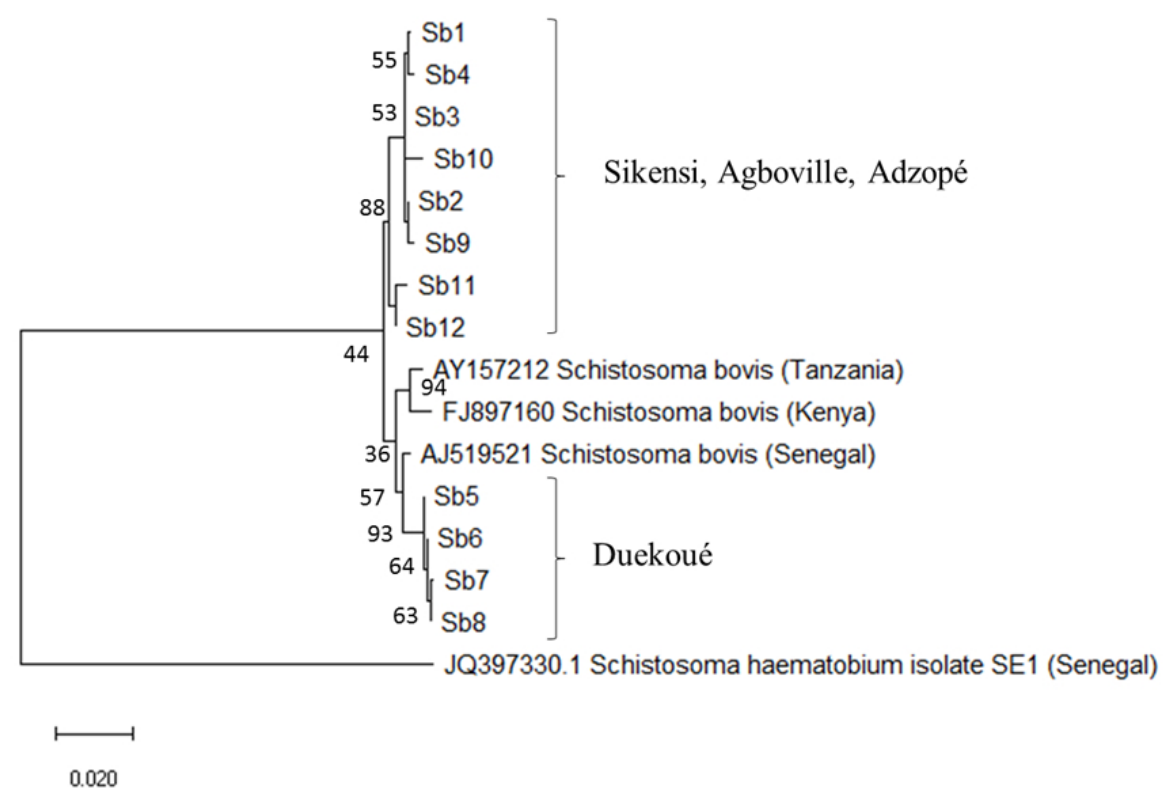

$254 \times 190 \mathrm{~mm}(96 \times 96 \mathrm{DPI})$ 
Table 1. Results of cox1-based rapid diagnostic (RD) PCR analysis of all miracidia collected per patient and of subsequent sequence analysis of a subsample. For RD-PCR analysis, the total number of miracidia, the number (and percentage) determined as Schistosoma haematobium cox1 and S. bovis cox 1 and the level of significance of differences between parasites per study area using Fisher's exact test are shown. For sequencing, 10 miracidia were randomly selected (five $S$. haematobium and five $S$. bovis) per study area, based on the mitochondrial-cox1 RD-PCR results. For sequence analysis, number analysed (n) and the times different combinations of cox1 haplotype and ITS2 alleles found are given together with the resulting species classification.

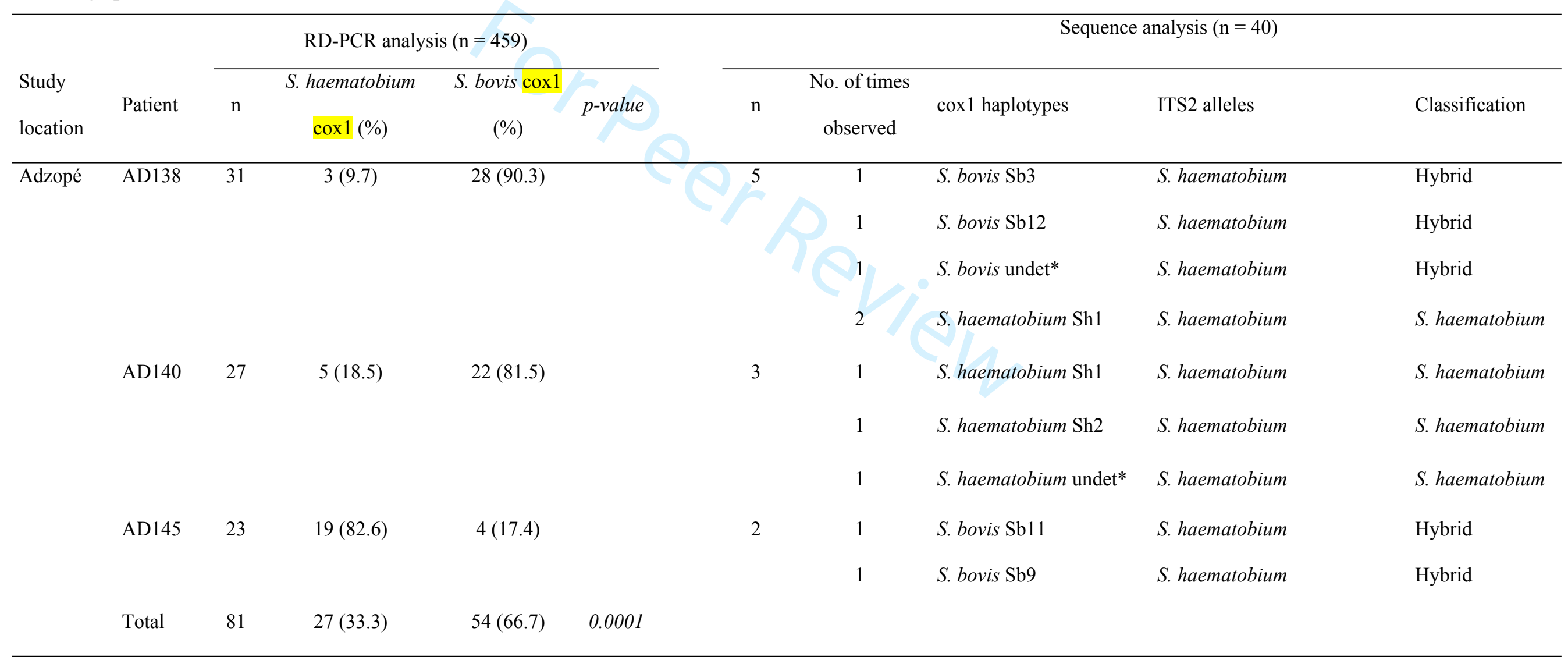




\begin{tabular}{|c|c|c|c|c|c|c|c|c|c|}
\hline \multirow[t]{10}{*}{ Agboville } & AG062 & 19 & $13(68.4)$ & $6(31.6)$ & 1 & 1 & S. bovis $\mathrm{Sb} 10$ & S. haematobium + S. bovis & Hybrid \\
\hline & AG068 & 23 & $23(100)$ & 0 & 3 & 2 & S. haematobium Sh3 & S. bovis & Hybrid \\
\hline & & & & & & 1 & S. haematobium Sh4 & S. bovis & Hybrid \\
\hline & AG077 & 24 & $3(12.5)$ & $21(87.5)$ & 3 & 1 & S. bovis $\mathrm{Sb} 3$ & S. haematobium + S. bovis & Hybrid \\
\hline & & & & & & 1 & S. bovis undet* & S. haematobium + S. bovis & Hybrid \\
\hline & & & & & & 1 & S. bovis $\mathrm{Sb} 9$ & S. bovis & S. bovis \\
\hline & AG108 & 13 & $9(69.2)$ & $4(30.8)$ & 2 & 1 & S. haematobium Sh6 & S. haematobium $+S$ bovis & Hybrid \\
\hline & & & & & & 1 & S. haematobium Sh5 & S. haematobium & S. haematobium \\
\hline & AG219 & 32 & $25(78.1)$ & $7(21.9)$ & 1 & 1 & S. bovis $\mathrm{Sb} 3$ & S. haematobium & Hybrid \\
\hline & Total & 111 & $73(65.8)$ & $38(34.2)$ & & & & & \\
\hline \multirow[t]{8}{*}{ Duekoué } & DU330 & 2 & $2(100)$ & 0 & 2 & 1 & S. haematobium Sh1 & S. haematobium & S. haematobium \\
\hline & & & & & & 1 & S. haematobium undet & S. haematobium & S. haematobium \\
\hline & DU337 & 35 & $35(100)$ & 0 & 2 & 2 & S. haematobium Sh1 & S. haematobium & S. haematobium \\
\hline & DU345 & 30 & $11(36.7)$ & $19(63.3)$ & 3 & 2 & S. bovis $\mathrm{Sb} 7$ & S. haematobium & Hybrid \\
\hline & & & & & & 1 & S. bovis Sb8 & S. haematobium & Hybrid \\
\hline & DU362 & 30 & $12(40.0)$ & $18(60.0)$ & 2 & 1 & S. bovis Sb5 & S. haematobium & Hybrid \\
\hline & & & & & & 1 & S. bovis Sb6 & S. haematobium & Hybrid \\
\hline & DU386 & 31 & $3(9.7)$ & $28(90.3)$ & 1 & 1 & S. haematobium Sh1 & S. haematobium & S. haematobium \\
\hline
\end{tabular}




\begin{tabular}{|c|c|c|c|c|c|c|c|c|c|c|}
\hline & Total & 128 & $63(49.2)$ & $65(50.8)$ & 0.721 & & & & & \\
\hline \multirow[t]{11}{*}{ Sikensi } & SI028 & 30 & $18(60.0)$ & $12(40.0)$ & & 2 & 1 & S. bovis $\mathrm{Sb} 3$ & S. haematobium & Hybrid \\
\hline & & & & & & & 1 & S. bovis $\mathrm{Sb} 4$ & S. haematobium & Hybrid \\
\hline & SI052 & 26 & $12(46.2)$ & $14(53.8)$ & & 2 & 1 & S. haematobium Sh7 & S. haematobium & S. haematobium \\
\hline & & & & & & & 1 & S. haematobium undet* & S. haematobium & S. haematobium \\
\hline & SI109 & 22 & $9(40.9)$ & $13(59.1)$ & & 1 & 1 & S. haematobium undet* & S. haematobium & S. haematobium \\
\hline & SI114 & 19 & $11(57.9)$ & $8(42.1)$ & & 1 & 1 & S. bovis $\mathrm{Sb} 3$ & S. haematobium & Hybrid \\
\hline & SI122 & 19 & $11(57.9)$ & $8(42.1)$ & & 2 & 1 & S. bovis $\mathrm{Sb} 2$ & S. haematobium + S. bovis & Hybrid \\
\hline & & & & & & & 1 & S. bovis $\mathrm{Sb} 1$ & S. haematobium & Hybrid \\
\hline & SI136 & 23 & $15(65.2)$ & $8(34.8)$ & & 2 & 1 & S. haematobium Sh1 & S. haematobium & S. haematobium \\
\hline & & & & & & & & S. haematobium undet* & S. haematobium & S. haematobium \\
\hline & Total & 139 & $76(54.7)$ & $63(45.3)$ & 0.026 & & & & & \\
\hline
\end{tabular}

Cox1, cytochrome oxidase subunit I gene; ITS, internal transcribed spacer region.

p-value $<0.05$ was considered significant

* undet., sequences for which the exact haplotype could not be determined due to sequence quality 


\section{Supplementary file 1}

Table S1. Nucleotide sequence at the five polymorphic sites within the $1200 \mathrm{bp}$ cox 1 sequences of the different $S$. haematobium haplotypes found, using haplotype Sh1 as reference. $n$, number of miracidia with the respective haplotypes.

\begin{tabular}{|c|c|c|c|c|c|c|}
\hline \multirow[t]{2}{*}{ Haplotype } & \multirow[t]{2}{*}{$\mathrm{n}$} & \multicolumn{5}{|c|}{ Position of polymorphic site } \\
\hline & & 13 & 18 & 66 & 635 & 998 \\
\hline Shl & 8 & $\mathrm{~T}$ & A & $\mathrm{T}$ & G & $\mathrm{T}$ \\
\hline $\operatorname{Sh} 2$ & 1 & & . & . & . & A \\
\hline $\operatorname{Sh} 3$ & 2 & G & . & $\mathrm{C}$ & . & . \\
\hline Sh4 & 1 & G & $\mathrm{T}$ & $\mathrm{C}$ & . & . \\
\hline $\operatorname{Sh} 5$ & 1 & . & & $\mathrm{C}$ & . & . \\
\hline Sh6 & 1 & G & & . & . & . \\
\hline $\operatorname{Sh} 7$ & 1 & . & & . & A & . \\
\hline
\end{tabular}




\section{Supplementary file 2}

Table S2. Nucleotide sequence at the 27 polymorphic sites within the $1200 \mathrm{bp}$ cox 1 sequences of the different $S$. bovis haplotypes found, using haplotype $\mathrm{Sb} 1$ as reference. $\mathrm{n}$, number of miracidia with the respective haplotype.

\section{Position of polymorphic site}

\begin{tabular}{|c|c|c|c|c|c|c|c|c|c|c|c|c|c|c|c|c|c|c|c|c|c|c|c|c|c|c|c|c|}
\hline Haplotype & $\mathrm{n}$ & \multicolumn{3}{|c|}{$\begin{array}{lll}106 & 108 & 132\end{array}$} & \multicolumn{2}{|c|}{$133 \quad 136$} & \multicolumn{3}{|c|}{$192 \quad 293 \quad 295$} & \multicolumn{2}{|c|}{319353} & \multicolumn{3}{|c|}{$355 \quad 616 \quad 666$} & \multicolumn{2}{|c|}{$685 \quad 712$} & \multicolumn{2}{|c|}{$\begin{array}{ll}763 \quad 829\end{array}$} & \multicolumn{2}{|c|}{$949 \quad 973$} & \multicolumn{2}{|c|}{976980} & \multicolumn{2}{|c|}{$\begin{array}{ll}982 & 994\end{array}$} & \multicolumn{2}{|c|}{$\begin{array}{ll}996 & 1000\end{array}$} & 1001 & 1008 \\
\hline$S b 1$ & 1 & $\mathrm{~T}$ & $\mathrm{~T}$ & G & $\mathrm{T}$ & G & A & A & A & $\mathrm{C}$ & $\mathrm{C}$ & $\mathrm{T}$ & $\mathrm{T}$ & $\mathrm{C}$ & $\mathrm{C}$ & G & $\mathrm{C}$ & $\mathrm{T}$ & G & $\mathrm{C}$ & $\mathrm{C}$ & $\mathrm{A}$ & $\mathrm{G}$ & $\mathrm{C}$ & $\mathrm{T}$ & $\mathrm{G}$ & $\mathrm{G}$ & A \\
\hline$S b 2$ & 1 & . & $\mathrm{C}$ & . & & . & $\mathrm{T}$ & $\mathrm{G}$ & & & & $\cdot$ & . & . & . & . & . & . & . & . & . & . & . & . & . & . & . & . \\
\hline$S b 3$ & 5 & . & $\mathrm{C}$ & . & . & . & $\cdot$ & G & & . & & & . & . & . & . & . & . & . & . & . & . & . & . & . & . & . & . \\
\hline$S b 4$ & 1 & . & $\mathrm{C}$ & A & G & . & & . & . & . & & & & & . & . & . & . & . & . & . & . & . & . & . & . & . & . \\
\hline Sb5 & 1 & . & $\mathrm{C}$ & . & . & A & . & G & G & $\mathrm{T}$ & $\mathrm{T}$ & G & . & $\mathrm{T}$ & $\mathrm{T}$ & A & $\mathrm{T}$ & $\mathrm{C}$ & A & A & . & G & A & $\mathrm{T}$ & . & . & . & . \\
\hline Sb6 & 1 & . & . & . & . & A & . & G & G & $\mathrm{T}$ & $\mathrm{T}$ & G & . & $\mathrm{T}$ & $\mathrm{T}$ & A & $\mathrm{T}$ & $\mathrm{C}$ & A & A & . & $\mathrm{G}$ & A & $\mathrm{T}$ & . & . & . & . \\
\hline$S b 7$ & 2 & $\mathrm{C}$ & . & . & . & A & $\mathrm{T}$ & G & G & $\mathrm{T}$ & $\mathrm{T}$ & G & . & $\mathrm{T}$ & $\mathrm{T}$ & A & $\mathrm{T}$ & C & A & A & . & $\mathrm{G}$ & A & $\mathrm{T}$ & . & . & . & . \\
\hline$S b 8$ & 1 & . & . & . & . & A & $\mathrm{T}$ & G & G & $\mathrm{T}$ & $\mathrm{T}$ & G & . & $\mathrm{T}$ & $\mathrm{T}$ & A & $\mathrm{T}$ & $\mathrm{C}$ & A & A & . & $\mathrm{G}$ & A & $\mathrm{T}$ & . & . & . & . \\
\hline$S b 9$ & 2 & C & . & . & . & . & $\mathrm{T}$ & G & . & . & . & . & . & . & . & . & . & . & . & . & . & . & . & . & . & . & . & . \\
\hline$S b 10$ & 1 & . & $\mathrm{C}$ & . & . & . & . & G & . & . & . & . & . & . & . & . & . & . & . & A & . & . & . & . & A & $\mathrm{C}$ & $\mathrm{C}$ & $\mathrm{C}$ \\
\hline$S b 11$ & 1 & $\mathrm{C}$ & . & . & . & . & $\mathrm{T}$ & G & . & . & . & . & $\mathrm{C}$ & . & $\mathrm{T}$ & . & $\mathrm{T}$ & . & A & A & $\mathrm{T}$ & . & . & . & . & . & . & . \\
\hline$S b 12$ & 1 & . & $\mathrm{C}$ & . & & . & & G & . & . & & . & $\mathrm{C}$ & . & $\mathrm{T}$ & . & $\mathrm{T}$ & . & A & A & $\mathrm{T}$ & . & & . & . & . & . & . \\
\hline
\end{tabular}

